\title{
GRACE score also predicts anatomic complexity of coronary artery disease patients presenting with non-STEMI
}

\author{
Umit Yasar Sinan, MD, Ozge Cetinarslan, MD, Veysel Oktay, MD, Alev Arat Ozkan, MD
}

\begin{abstract}
Sinan UY, Cetinarslan O, Oktay V, et al. GRACE score also predicts anatomic complexity of coronary artery disease patients presenting with non-STEMI. Curr Res Cardiol. 2017;4(3):52-54.

ABSTRACT

Acute Coronary Syndromes (ACS) represent the most common cause of death in the western world. We retrospectively screened data of non-STEMI patients admitted to the coronary care unit of a tertiary center between March 2015 and March 2016. GRACE score was calculated and patients were classified into low (1 to 108), intermediate (109 to 140) and high risk (>40) groups according to GRACE categories. SYNTAX scores were also calculated. 201 patients (mean age: $63 \pm 12$ years, 53.7\% female) were admitted with a diagnosis of non-STEMI. The mean GRACE score and SYNTAX score of study population were $105 \pm 34.1$ and $16.9 \pm 12$. Based on the GRACE score for in-hospital deaths, the SYNTAX score was $14.2 \pm 10.1$ in the low-risk group,
\end{abstract}

$16.0 \pm 13.4$ in the intermediators group, and $24 \pm 12.2$ in high-risk group (ANOVA $\mathrm{p}<0.0001$ ). Post-hoc Tukey analysis showed that the high-risk group had a significantly higher SYNTAX score than the low-risk and intermediate risk groups $(\mathrm{p}<0.0001 \mathrm{vs} p=0.003$ respectively). There were significant positive correlations between the SYNTAX score and GRACE scores of the study population calculated at admission for in-hospital deaths $(\mathrm{r}=0.363, \mathrm{p}<0.0001)$. GRACE score can predict complexity of CAD (high risk coronary anatomy). As we can decide to perform early invasive strategy according to GRACE score, we may consider detecting high risk complex coronary anatomy during coronary angiography. So, we may be ready to discuss with heart team about treatment strategy (ad hoc-PCI, multi-vessel PCI or CABG) in patients with high GRACE score. Before giving ADP receptor antagonist, we may consider CABG requirement in these patient population.

Key Words: GRACE score; SYNTAX score; Risk evaluation; Coronary artery disease; Non-STEMI

\begin{abstract}
A cute coronary syndromes (ACS) represent the most common cause A of death in the western world (1). The term ACS defines a large spectrum of patients including unstable angina, myocardial infarction without ST segment elevation (non-STEMI) and myocardial infarction with ST segment elevation (STEMI) all of which have different mortality and morbidity risks, thus different treatment strategies are applied. Primary percutaneous coronary intervention (PCI) is the treatment of choice for STEMI with a Class I indication whereas for non-STEMI current cardiology guidelines recommend a risk based treatment approach so that time and use of certain pharmacological and interventional strategies are decided according to patient's risk status (2-5). The most widely used risk scores are the "Thrombolysis in Myocardial Infarction (TIMI) and 'Global Registry of Acute Coronary Events' (GRACE) risk scores and their prognostic value has been validated in different clinical trials $(6,7)$. GRACE score has been shown a better predictor of clinical outcome than the TIMI score $(8,9)$. Both of these risk scores include only clinical variables whereas not only data derived from clinical characteristics but also anatomic extent and severity of the disease contributes significantly to the patient's risk and has an important impact on treatment decision. $40 \%$ to $60 \%$ of STEMI patients have multi-vessel disease (10). The risk of mortality and re-infarction are much higher among patients with multi-vessel disease than single vessel disease (11). Although the risk is high in this group, no randomized data exist about the role of complete coronary revascularization in both NSTEMI and STEMI patients. In this setting, guidelines are also ambiguous and inconclusive $(3,4)$.
\end{abstract}

The SYNergy between coronary intervention with TAXus and cardiac surgery (SYNTAX) I score is validated as a comprehensive tool for grading the complexity of Coronary Artery Disease (CAD) as well as discriminating low and high-risk patients for percutaneous or surgical intervention in patients with multi-vessel disease (12). Current guidelines recommend SYNTAX I score to identify the potential candidates for surgery among patients with ACS. Especially among those with non-STEMI before invasive imaging is of utmost importance as clopidogrel use prior to surgery is a major concern in this group.

This study is designed to assess the usefulness of GRACE risk score on admission in predicting high risk anatomy of non-STEMI patients defined by angiographic SYNTAX I score.

\section{SUBJECTS AND METHODS}

In this single center study, we retrospectively screened data of non-STEMI patients admitted to the coronary care unit of a tertiary center between March
2015 and March 2016. Records of patients with a diagnosis of non-STEMI were re-evaluated to confirm the diagnosis. Non-STEMI was defined as new onset or worsening chest pain occurring at rest or with minimal exertion with positive cardiac markers (troponin value above the $99^{\text {th }}$ percentile) and without ST segment elevation (3-5). Patients who is not suitable coronary angiography during index admission (due to death, patient rejection, physician preference), patients with previous coronary artery bypass grafting $(\mathrm{CABG})$ and PCI history, malignancy, valvular heart disease (especially severe aortic stenosis), hypertrophic cardiomyopathy, inflammatory disorders and conditions causing troponin elevation other than coronary artery disease were excluded. Clinical, biochemical and angiographic findings were obtained from hospital records. All electrocardiograms (ECG) were reevaluated to confirm the diagnosis.

GRACE score consisting of eight variables (age, heart rate on admission, systolic blood pressure (SBP) on admission, plasma creatinine, Killip class, ST-segment deviation, elevated troponins and cardiac arrest at admission) was calculated as described ranging from 0 to 372 (8). Patients were classified into low (1-108), intermediate (109-140) and high risk $(>140)$ groups according to GRACE categories.

\section{Coronary angiography and SYNTAX I score}

In our institution, selective coronary angiograms are performed using standard projections, two for right coronary artery (RCA) (left anterior oblique-LAO $30^{\circ}$ and anteroposterior-AP Cranial $15^{\circ}$ to $20^{\circ}$ ) and five for left anterior descending artery (LAD) (AP Caudal $15^{\circ}$ to $20^{\circ}$, Left $40^{\circ}$ and Caudal $20^{\circ}$, Left $40^{\circ}$ and Cranial $20^{\circ}$, AP Cranial $40^{\circ}$ and Left $40^{\circ}$ and Caudal $20^{\circ}$ ). Coronary angiograms were evaluated by two senior invasive cardiologists who were used to SYNTAX I scoring algorithm and blinded to patient's clinical data. SYNTAX I scores were calculated as described (12) and patients were also grouped as having 1-, 2- or 3-vessel disease. Stenosis $>50 \%$ were considered as significant.

The study protocol was approved by the Istanbul University Institute of Cardiology Ethics Committee.

\section{STATISTICAL METHOD}

Continuous variables are presented as mean \pm standard deviation and were compared using the Student-t test. Categorical variables are summarized as numbers and percentages and were compared by the Pearson's Chi-square test. The associations between the SYNTAX I score and patient characteristics

Department of Cardiology, Istanbul University Institute of Cardiology, Turkey

Correspondence: Dr Umit Yasar Sinan, MD, Department of Cardiology, Istanbul University Institute of Cardiology, Turkey, Telephone 90(533)396-8403, fax 90(212)459. 2021, e-mail drumityasar@hotmail.com

Received: August 09, 2017, Accepted: September 04, 2017, Published: September 06, 2017

OPEN $\bigcirc$ Access

This open-access article is distributed under the terms of the Creative Commons Attribution Non-Commercial License (CC BY-NC) (http:// creativecommons.org/licenses/by-nc/4.0/), which permits reuse, distribution and reproduction of the article, provided that the original work is properly cited and the reuse is restricted to noncommercial purposes. For commercial reuse, contact reprints@pulsus.com 
or laboratory parameters were assessed by Pearson or Spearman correlation tests. To assess the association between the GRACE score and the SYNTAX I score, some analyses were used. First, the linear association of each risk score with the SYNTAX I score was assessed by use of Pearson's correlation test. Second, ANOVA with the post-hoc Tukey test was used to compare SYNTAX I score values between low-, intermediate-, and high-risk groups based on the GRACE score for death in the hospital. Third, the receiver-operating characteristics (ROC) curve was used to test the predictive accuracy of the risk scores with respect to the presence of high risk $(\geq 140)$, intermediate risk $(109-140)$ or low risk $(\leq 108)$ based on the GRACE score. Significant prediction was accepted when the area under the ROC curve was significantly different from 0.5 . A p value $<0.05$ was accepted as statistically significant. All analyses were performed with SPSS 21.0 for Windows.

\section{RESULTS}

During the study period, 201 patients (mean age: 63 years \pm 12 years, $53.7 \%$ female) were admitted to our coronary unit with a diagnosis of non-STEMI. More than half of the patients had diabetes mellitus (63.7\%), previous CAD history $(74.6 \%)$ and familial history of CAD (72.1\%). The mean GRACE score, SYNTAX I score and CRUSADE score of study population were 105 $\pm 34.1,16.9 \pm 12.1$ and $27.7 \pm 15.0$ respectively. Clinical characteristics and laboratory values of the study population are presented in the Table 1.

Based on the GRACE score for in-hospital deaths, 108 (53.7\%) patients were included in the low-risk group, $44(21.9 \%)$ in the intermediators group, and 49 (24.4\%) in the high-risk (141-372) group. The ratio of low risk and intermediate-high risk patients (according to GRACE score) in study population were nearly similar $(53.7 \%$ and 46.3$)$. The analysis of coronary angiography showed that the mean SYNTAX I score of the study population was $16.9 \pm 12.1$. Based on the GRACE score for in-hospital deaths, the SYNTAX I score was $14.2 \pm 10.1$ in the low-risk group, $16.0 \pm 13.4$ in the intermediators group, and $24 \pm 12.2$ in high-risk group (ANOVA, $p<0.0001$ ) (Figure 1).

Post-hoc Tukey analysis showed that the high-risk group had a significantly higher SYNTAX I score than the low-risk and intermediate risk groups $(\mathrm{p}<0.0001$ vs $\mathrm{p}=0.003$ respectively). There were significant positive correlations between the SYNTAX I score and GRACE scores of the study population calculated at admission for in-hospital deaths $(\mathrm{r}=0.363, \mathrm{p}<0.0001)$ (Figure 2).

The GRACE score of the study population calculated at admission for inhospital deaths showed good discriminatory capacity between the patients with and without a high-risk (>33) SYNTAX I score, with an area under the ROC curve of 0.644 (CI 0.567-0.721, p<0.0001) (Figure 3).

\section{TABLE 1}

Demographic and clinical characteristics and laboratory values of the study group

\begin{tabular}{cc}
\multicolumn{2}{c}{ Study group (n:201) } \\
Age, years & $63.3 \pm 12.4$ \\
Female, (\%) & 53.7 \\
Diabetes mellitus (\%) & 63.7 \\
Hypertension, (\%) & 36.8 \\
Hypercholesterolemia (\%) & 33.3 \\
Smoking (\%) & 50.2 \\
Family history (\%) & 72.1 \\
CAD history (\%) & 74.6 \\
CRUSADE score & $27.7 \pm 15.0$ \\
GRACE score & $105.0 \pm 34.1$ \\
SYNTAX I score & $16.9 \pm 12.1$ \\
EF (\%) & $53.4 \pm 9.8$ \\
Laboratory values & \\
Basal creatinine, mg/dL & $0.92 \pm 0.31$ \\
eGFR, mL/min/1.73 m ${ }^{2}$ & $87.7 \pm 29.0$ \\
BUN, mg/dL & $17.9 \pm 9.5$ \\
Glucose, mg/dL & $130.1 \pm 55.1$ \\
Total cholesterol, mg/dl & $187.6 \pm 41.6$ \\
LDL, mg/dL & $127.7 \pm 35.6$ \\
HDL, mg/dL & $38.3 \pm 13.1$ \\
Triglyceride, mg/dL & $167.4 \pm 107.5$
\end{tabular}

BUN: Blood Urea Nitrogen; CAD: Coronary Artery Disease; CABG: Coronary Artery By-pass Grafting; Egfr: Estimated Glomerular Filtration Rate; HDL: High Density Lipoprotein;LDL: Low Density Lipoprotein

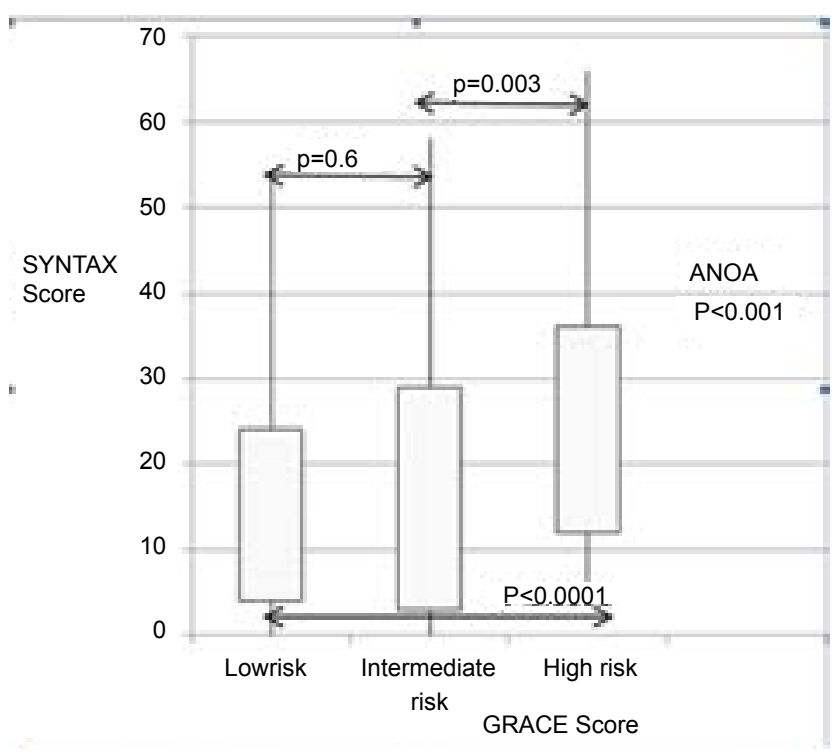

Figure 1) The comparison of SYNTAX I score between risk groups

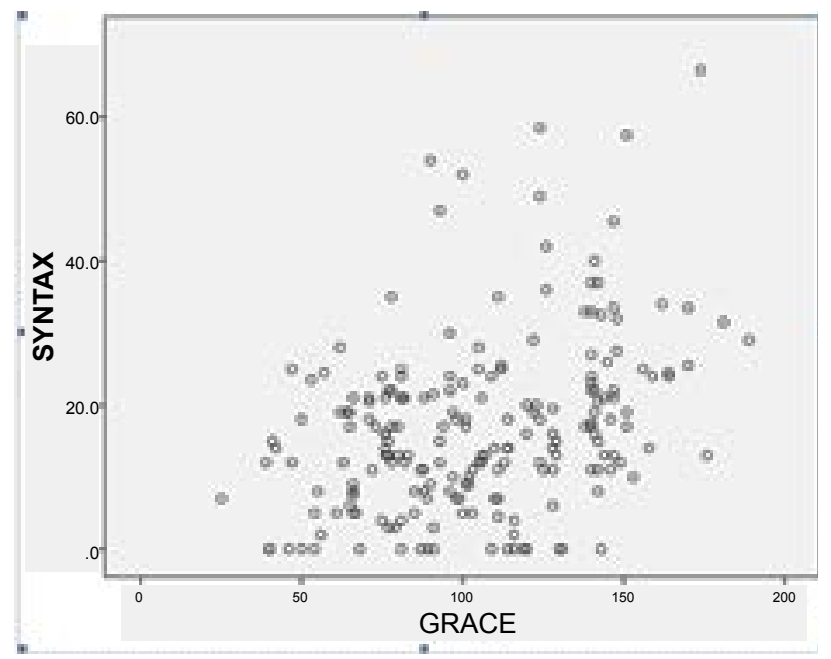

Figure 2) The correlation between the GRACE score and the SYNTAX I score

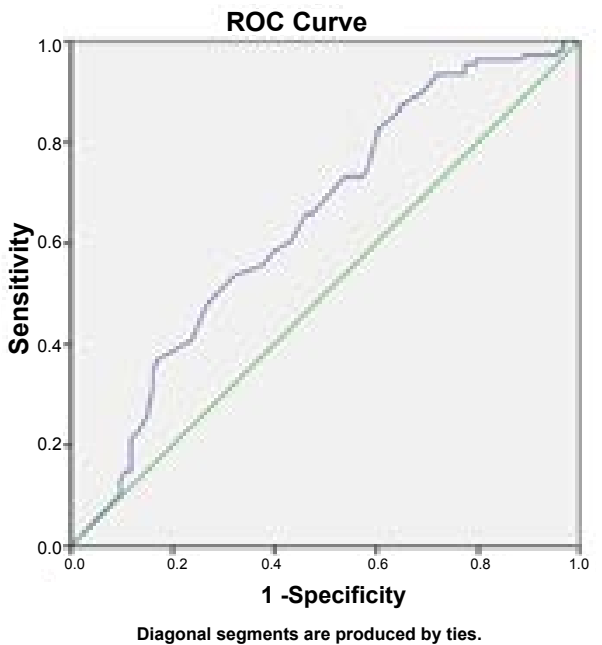

Figure 3) The ROC curve of the GRACE score for detecting SYNTAX I Score (the area under the ROC curve of 0.644 (CI 0.567-0.721, $p<0.0001$ ))

\section{DISCUSSION}

The GRACE scoring system is currently used for risk stratification of patients with non-STEMI. It is based on multivariable models that integrate elements from the medical history, physical examination, presence of cardiac arrest on admission, ST-T changes on admission ECG, and biochemical evidence of 
myocyte necrosis (8). Given the wide spectrum of risk for death and recurrent events among patients with NSTE-ACS, the latest ESC guideline of 2015 recommends an early invansive strategy ( $<24$ hours) in non-STEMI patients of high risk (GRACE score >140) (Class IB) (5). Our study population was consisted of $46.3 \%$ intermediate-high risk patients according to GRACE score.

GRACE score alone is a clinical score. It identifies the high-risk patients that may benefit from early invasive strategy (8). Nevertheless, the prognosis of the patients cannot be considered independently of the extent and complexity of CAD. In this study, we used SYNTAX I score, a quantitative angiographic score, to determine the severity of atherosclerotic burden, as the latest ESC guideline recommends (5). The SYNTAX I score is widely accepted and its prognostic value has been demonstrated (12). SYNTAX I score $\geq 33$ shows a highly complex CAD, and current European re-vascularization guidelines recommends surgical re-vascularization for subjects with a SYNTAX I score of $\geq 33$ (class IA) $(4,5)$. The mean SYNTAX I score of our study population was $16.9 \pm 12.1$

The latest ESC guideline of 2015 also states to base the revascularization strategy (e.g. ad hoc culprit-lesion PCI, multi-vessel PCI, CABG) on the clinical status and comorbidities as well as the disease severity (including distribution, angiographic lesion characteristics, SYNTAX I score), according to the local Heart Team protocol (Class IC) (5). Our study revealed that, in patients with non-STEMI, the GRACE score has a positive predictive value for the presence of a greater extent and burden of CAD and most likely requiring CABG. The positive relationship between the GRACE and SYNTAX I scores may have some clinical implications. As we can determine the group of patients that benefit from early invasive strategy by calculating the GRACE score on admission, we can also predict the complexity of CAD before coronary angiography through this positive correlation between GRACE and SYNTAX I score. Predicting high risk coronary anatomy before coronary angiography is quite important because it affects the treatment modality (e.g. ad hoc culprit-lesion PCI, multi-vessel PCI, or CABG) and antiplatelet choice (to prevent CABG related major bleeding).

There are studies that investigate the role of GRACE score in the prediction of high-risk coronary anatomy in patients with NSTE-ACS in the literature. Kilickiran et al. demonstrated association between high risk coronary anatomy and GRACE score (13). In their study, patients with high risk coronary anatomy (based on SYNTAX I score $\geq 33$ ) had higher GRACE score than low risk coronary anatomy (SYNTAX I score <33). Differently we used GRACE score to predict complexity of CAD in our study. Patients were divided into 3 groups (low, intermediate and high risk) according to GRACE score in our study and the SYNTAX I score was the highest in the high-risk population (GRACE score $>140$ ). So, we demonstrated that a clinical risk score can predict anatomic complexity. So, we may advice against giving ADP receptor antagonist (clopidogrel, ticagrelor) before coronary angiography to consider $\mathrm{CABG}$ requirement according to our study results. But our study is small sample size, single center experience and retrospective in nature. We need large scale and prospective studies to be able to give such important recommendations.

\section{CONCLUSION}

In conclusion, GRACE score can predict complexity of CAD (high risk coronary anatomy). As we can decide to perform early invasive strategy according to GRACE score, we may consider detecting high risk complex coronary anatomy during coronary angiography. So, we may be ready to discuss with Heart team about treatment strategy (ad hoc-PCI, multi-vessel $\mathrm{PCI}$ or $\mathrm{CABG}$ ) in patients with high GRACE score. Before giving ADP receptor antagonist, we may consider $\mathrm{CABG}$ requirement in these patient population.

\section{CONFLICT OF INTEREST}

The authors declare that they have no conflict of interest.

\section{REFERENCES}

1. Murray CJ, Lopez A. Mortality by cause for eight regions of the world: Global burden of disease study. Lancet. 1997;349:1269-76.

2. O'Gara PT, Kushner FG, Ascheim DD, et al. 2013 ACCF/AHA guideline for the management of ST-elevation myocardial infarction: A report of the American College of Cardiology Foundation/American Heart Association task force on practice guidelines. Circulation. 2013;127:e362-425.

3. Amsterdam EA, Wenger NK, Brindis RG, et al. ACCF/AHA Guideline for the management of patients with non-ST-elevation acute coronary syndromes: A report of the American College of Cardiology Foundation/American Heart Association task force on practice guidelines. Circulation. 2014;130:344-426.

4. Windecker S, Kolh P, Alfonso F, et al. 2014 ESC/EACTS Guidelines on myocardial revascularization. The task force on myocardial revascularization of the European Association of Percutaneous Cardiovascular Intervention (EAPCI). Eur Heart J. 2014;35:2541-619.

5. Roffi M, Patrono C, Collet JP, et al. 2015 ESC guidelines for the management of acute coronary syndromes in patients presenting without persisten ST segment elevation. Eur Heart J. 2015;37(3):267-315.

6. Antman EM, Cohen M, Bernink PJ, et al. The TIMI risk score for unstable angina/non-ST elevation MI: A method for prognostication and therapeutic decision making. JAMA 2000;284:835-42.

7. Granger $\mathrm{CB}$, Goldberg RJ, Dabbous $\mathrm{O}$, et al. Global registry of acute coronary events investigators. Predictors of hospital mortality in the global registry of acute coronary events. Arch Intern Med. 2003; 163:2345-53

8. De Araújo Gonçalves P, Ferreira J, Aguiar C, et al. TIMI, PURSUIT, and GRACE risk scores: Sustained prognostic value and interaction with revascularization in NSTE-ACS. Eur Heart J. 2005;26:865-72.

9. Ramsay G, Podogrodzka M, McClure C, et al. Risk prediction in patients presenting with suspected cardiac pain: The GRACE and TIMI risk scores versus clinical evaluation. QJM. 2007;100:11-8.

10. Park DW, Clare RM, Schulte PJ, et al. Extent, location, and clinical significance of non-infarct-related coronary artery disease among patients with ST elevation myocardial infarction. JAMA. 2014;312:2019.

11. Halkin A, Singh M, Nikolsky E, et al. Prediction of mortality after primary percutaneous coronary intervention for AMI: The CADILLAC risk score. J Am Coll Cardiol. 2005;45:1397.

12. Sianos G, Morel MA, Kappetein AP, et al. The SYNTAX score: An angiographic tool grading the complexity of coronary artery disease. Euro Intervention. 2005;1:219-27.

13. Avci BK, Ikitimur B, Tok OO, et al. The role of GRACE score in the prediction of high-risk coronary anatomy in patients with non-ST elevation acute coronary syndrome. Kardiologia Polska. 2015;73:592-7. 